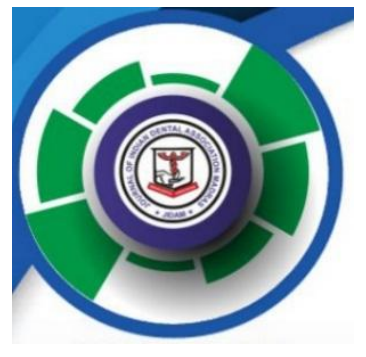

ORIGINAL ARTICLE

\title{
KNOWLEDGE, ATTITUDE AND PRACTICES OF DENTAL STUDENTS AND PRACTITIONERS TOWARDS CONSCIOUS SEDATION - A CROSS-SECTIONAL STUDY
}

\section{Dr.Vinita Mary. A, Dr. Kesavan. R, Dr. Keerthiga. B, Dr. Neelofar Begum.A , Dr. Sathya.K, Dr. Malleswari.M.D}

Department of Public Health Dentistry, Thai Moogambigai Dental College and Hospital, Chennai, Tamil Nadu, India.

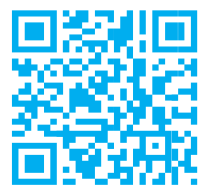

DOI: $10.37841 /$ jidam_2021_V8_I3_01

Address for Correspondence

Dr. Vinita Mary. A, MDS

Professor and Head,

Department of Public Health Dentistry,

Thai Moogambigai Dental College

and Hospital, Chennai, Tamil

Nadu, India.

Email id: viniebe@gmail.com

Received: 12.08.2021 First Published: 14.09.2021

Accepted: 11.09.2021

Published: 27.09.2021

\begin{abstract}
BACKGROUND: Dental professionals work effectively and efficiently to impart a positive behavioral attitude towards dental treatment in children with oral health care needs. The main objective of sedation in pediatric dentistry is to reduce fear, minimize physical discomfort and control behavior for dental procedures. The present study was carried out to evaluate the knowledge, attitude and practices of dental students and practitioners towards conscious sedation.
\end{abstract}

METHODS: A descriptive cross-sectional survey was conducted among 384 dental professionals that included undergraduate, postgraduate dental students and dental practitioners across Tamil Nadu, India. The data was collected through self-constructed questionnaires consisting of 28 questions in English and circulated via online platform on account of COVID-19 pandemic. Every respondent was informed of the purpose of the study and participation was purely voluntary.

RESULTS: The mean age of the study participants was found to be $25.99 \pm$ 12.34 years (mean \pm S.D) comprising of 184 (48\%) males and $200(52 \%)$ females. Majority of the study participants acquired knowledge about conscious sedation through dental curriculum only. About $46.1 \%$ considered conscious sedation over general anesthesia for all dental procedures like restoration, scaling, minor bone fractures and minor surgeries, $40.1 \%$ preferred midazolam and $78.4 \%$ recommend Nitrous oxide sedation as effective technique. Most of the study participants believed oral sedation was easier to administer, required less expertise and skill with minimal or no complication.

CONCLUSION: This study reveals lacunae in adequate knowledge and understanding among the practitioners. The result of this study thus provides an insight on need for strategies like incorporating guidelines in dental curriculum, organizing continuing dental education programs and workshops to provide knowledge and incorporate positive attitude among the dental professionals to offer effective conscious sedation in children necessitating oral health care.

KEYWORDS: Dental anxiety, Midazolam, Pediatric dentistry, general 
Vinita Mary et al: Knowledge, Attitude And Practices Of Dental Students And Practitioners

\section{INTRODUCTION:}

Management of oral health care needs among children is a demanding task owing to several factors such as anxiety, fear associated with anticipation of pain and agespecific behavioral patterns ${ }^{1,2}$. The process by which dental health care professionals work effectively and efficiently to impart positive behavior attitude towards dental treatment in children is often termed as behavior management ${ }^{3}$. Behavioral modification during dental treatment can be achieved by non-pharmacological methods like communication techniques, contingency management, coping, assimilation, behavioral shaping and modeling methods ${ }^{4}$ as well as pharmacological methods such as premedications, deep sedations, conscious sedations, general anesthesia and anxiolytic or minimal sedation methods ${ }^{5,6}$. The AAPD (American Academy of Pediatric Dentistry) recommends use of general anesthesia or conscious sedation techniques in acute anxiety situation, extensive dental rehabilitation procedure of longer duration, severe treatment procedural situations, extremely uncooperative child behavior, and an immature cognitive pattern in a child or in case of children with physical or physiological impairments ${ }^{7,8}$. Thus the main objective of sedation in pediatric dentistry is to reduce fear, minimize physical discomfort and control behavior for dental procedures.

General anesthesia is preferred in children less than 4 years of age, patients with special health care needs, medically compromised conditions including respiratory illness, hepatic and renal diseases, epilepsy and with communicating incapability. However, the major drawback of this technique includes prolonged unconsciousness, depressed vital signs and protective reflexes and need for specific equipment and well trained anesthetic team ${ }^{9,10,11 .}$ In order to overcome these serious complications, conscious sedation was introduced to perform high quality dental care treatment with use of drugs that produce a state of CNS (Central nervous system) depression with maintained verbal contact through the entire treatment period of sedation. Conscious sedation is commonly preferred to reduce anxiety, increase pain threshold, to maintain stable vitals and reflexes and to achieve mood alteration. It is indicated in patients with severe dental anxiety and phobia, stress inducing medical conditions, children lacking psychological or emotional maturity requiring prolonged traumatic dental procedures ${ }^{12,13}$ but contraindicated in uncooperative and medically compromised children ${ }^{14,15}$. It is highly suggested that pediatric dental professionals and dental students should have a comprehensive theoretical knowledge on anatomic and physiological differences in children, prerequisites for conscious sedation with some practical understanding of patient selection, assessment and preparation with familiarity towards various sedation techniques such as inhalation, oral, intramuscular, submucosal and several other recommended modes of administration as required. Thus, the present study was carried out to evaluate the knowledge, attitude and practices of undergraduate and post graduate dental students and dental practitioners towards conscious sedation.

\section{METHODOLOGY:}

A cross-sectional questionnaire survey was conducted among 384 dental professionals that included undergraduate and postgraduate dental students and dental practitioners across Tamil Nadu, India.

\section{ETHICAL CLEARANCE}

The study was conducted following the Helsinki declaration as revised in 2013. The protocol was submitted to the ethical committee before the start of the study and approval was obtained from Institutional Review Board.

\section{QUESTIONNAIRE DEVELOPMENT:}

The required information was collected through published scientific articles pertaining to the study and specially designed structured questionnaire, comprising of 28 questions divided into 2 sections was prepared. The first section was used to record the demographic details such as age, gender, qualification and year of study/experience of practice. The second section consisted a combination of selected responses to certain questions and also few close ended questions (Yes / No/ don't know) related to knowledge, attitude and practices towards conscious sedation.

\section{SAMPLING METHODOLOGY AND DATA COLLECTION}

A non-probabilistic convenient sampling methodology was used to recruit the participants for the present study. Since this study was conducted during COVID-19 Pandemic lockdown period, online Google forms were generated and distributed through social media platforms. The questionnaire encompassed a separate section which explained the nature and purpose of the study along with an informed consent form. The respondents were requested to provide appropriate answers and were assured of anonymity and confidentiality. The filled questionnaire was collected and evaluated. 
Vinita Mary et al: Knowledge, Attitude And Practices Of Dental Students And Practitioners

\section{STATISTICAL ANALYSIS}

The data was analyzed using Statistical Package for Social Sciences, IBM Corporation, SPSS Inc., Chicago, IL, USA Version 21 software package (SPSS). Descriptive analysis with frequency, percentage, mean and standard deviation was computed. Pearson Chi-square test and Fisher's Exact test was used to assess the level of significance at $\mathrm{p}<.05$.

\section{RESULTS}

The age of the participants in the present study ranged between 19 to 50 years with a mean age of $25.99 \pm 12.34$ years (mean \pm S.D) comprising of $184(48 \%)$ males and 200(52\%) females. Among the study participants, 89(23\%) were BDS practitioners, 55(14\%) were MDS practitioners, 178(47\%) were UG students and 62(16\%) were PG students. Around $70(18 \%)$ were studying in third year, 45 (12\%) were studying in final year, $83(22 \%)$ were interns, 61(16\%) were Post-graduate students, $80(21 \%)$ were dental practitioners under 5 years of experience, $31(8 \%)$ were practitioners with 5-10 years of experience, and 14(3\%) were practitioners with more than 10years of experience. The demographic details of the study participants is given in table 1.

\begin{tabular}{|l|l|l|}
\hline $\begin{array}{c}\text { Demographic } \\
\text { character }\end{array}$ & MEAN & \\
\hline \multirow{2}{*}{ AGE } & SD & 25.99 \\
\hline & & 12.339 \\
\hline \multirow{2}{*}{ GENDER } & MALE & $\mathrm{N}(\%)$ \\
\cline { 2 - 3 } & FEMALE & $184(48 \%)$ \\
\hline \multirow{2}{*}{ QUALIFICATION } & BDS & $200(52 \%)$ \\
\cline { 2 - 3 } & MDS & $89(23 \%)$ \\
\hline
\end{tabular}

\begin{tabular}{|l|l|l|}
\hline \multirow{5}{*}{$\begin{array}{l}\text { YEAR OF STUDY } \\
\text { OF PRACTIENCE }\end{array}$} & UG STUDENTS & $178(47 \%)$ \\
\cline { 2 - 3 } & PG STUDENTS & $62(16 \%)$ \\
\cline { 2 - 3 } & FINALD YEAR & $70(18 \%)$ \\
\cline { 2 - 3 } & INTERN & $45(12 \%)$ \\
\cline { 2 - 3 } & POST GRADUATE & $83(22 \%)$ \\
\cline { 2 - 3 } & $\begin{array}{l}\text { DENTAL } \\
\text { PRACTIONERS UNDER } \\
\text { 5 EXPERIENCE }\end{array}$ & $80(21 \%)$ \\
\cline { 2 - 3 } & $\begin{array}{l}\text { 5-10 YEARS OF } \\
\text { EXPERIENCE }\end{array}$ & $31(8 \%)$ \\
\cline { 2 - 3 } & $\begin{array}{l}\text { MORE THEN 10 YEARS } \\
\text { OF EXPERIENCE }\end{array}$ & $14(3 \%)$ \\
\hline
\end{tabular}

Table 1: Demographic details of the study participants

When the genders were compared, it was observed that there were differences with respect to conscious sedation seminar attendance and monitoring of intravenous conscious sedation and these differences were statistically significant ( $\mathrm{p}<0.05)$ (Table 2 (a) and (b)).

The comparison of knowledge about conscious sedation based on educational qualification among the participants revealed that there was statistically significant differences for source of learning, alternative names, frequently used technique in dentistry, the fastest acting drug, stage of conscious sedation for performing dental treatment, indication, duration, period of pre-operative fasting and complications of conscious sedation $(\mathrm{p}<0.05)$ (Table 2 (a) and (b)).

\begin{tabular}{|c|c|c|c|c|c|c|c|c|c|c|}
\hline \multirow[t]{2}{*}{ Question } & \multirow[t]{2}{*}{ Options } & \multirow{2}{*}{$\begin{array}{l}\text { Total } \\
\text { n(\%) }\end{array}$} & \multicolumn{2}{|c|}{ Gender } & \multirow{2}{*}{$\begin{array}{c}P \\
\text { value }\end{array}$} & \multicolumn{4}{|c|}{ Qualification } & \multirow{2}{*}{$\begin{array}{c}\mathbf{P} \\
\text { value }\end{array}$} \\
\hline & & & $\begin{array}{l}\text { Males } \\
\text { n (\%) }\end{array}$ & $\begin{array}{c}\text { Females } \\
\text { n }(\%)\end{array}$ & & $\begin{array}{c}\text { BDS } \\
\text { n }(\%)\end{array}$ & $\begin{array}{l}\text { MDS } \\
\text { n }(\%)\end{array}$ & $\begin{array}{c}\text { UG student } \\
\text { n }(\%)\end{array}$ & $\begin{array}{c}\text { PG student } \\
\text { n }(\%)\end{array}$ & \\
\hline \multirow{4}{*}{$\begin{array}{l}\text { How did you } \\
\text { learn about } \\
\text { conscious } \\
\text { sedation }\end{array}$} & Seminar & $118(30.7 \%)$ & $55(29.9 \%)$ & $63(31.5 \%)$ & \multirow{4}{*}{0.725} & $29(32.6 \%)$ & $16(29.1 \%)$ & $56(31.5 \%)$ & $17(27.4 \%)$ & \multirow{4}{*}{0.025} \\
\hline & $\begin{array}{l}\text { Dental } \\
\text { curriculum }\end{array}$ & $195(50.8 \%)$ & $95(51.1 \%)$ & $100(50.0 \%)$ & & $54(60.7 \%)$ & $30(54.4 \%)$ & $77(43.3 \%)$ & $34(54.8 \%)$ & \\
\hline & Colleague & $58(15.1 \%)$ & $26(14.1 \%)$ & $32(16.0 \%)$ & & $4(4.5 \%)$ & $9(16.4 \%)$ & $35(19.7 \%)$ & $10(16.1 \%)$ & \\
\hline & Don't know & $13(3.4 \%)$ & $8(4.3 \%)$ & $5(2.5 \%)$ & & $2(2.2 \%)$ & $0(0.0 \%)$ & $10(5.6 \%)$ & $1(1.6 \%)$ & \\
\hline \multirow[b]{2}{*}{$\begin{array}{l}\text { Have you } \\
\text { attended any } \\
\text { seminar } \\
\text { regarding } \\
\text { conscious } \\
\text { sedation }\end{array}$} & Yes & $219(57.0 \%)$ & $115(62.5 \%)$ & $104(52.0 \%)$ & \multirow[b]{2}{*}{0.038} & $53(59.6 \%)$ & $44(80.0 \%)$ & $81(45.5 \%)$ & $41(66.1 \%)$ & \multirow[b]{2}{*}{0.000} \\
\hline & No & $165(43.0 \%)$ & $69(37.5 \%)$ & $96(48.0 \%)$ & & $36(40.4 \%)$ & $11(20.0 \%)$ & $97(54.5 \%)$ & 21(33.9) & \\
\hline \multirow{3}{*}{$\begin{array}{l}\text { What are the } \\
\text { other names for } \\
\text { conscious } \\
\text { sedation }\end{array}$} & $\begin{array}{l}\text { Sleep } \\
\text { dentistry }\end{array}$ & $118(30.7 \%)$ & $57(14.8 \%)$ & $61(30.5 \%)$ & \multirow{3}{*}{0.714} & $27(30.3 \%)$ & $5(9.1 \%)$ & $73(41.0 \%)$ & $13(21.0 \%)$ & \multirow{3}{*}{0.000} \\
\hline & $\begin{array}{l}\text { Twilight } \\
\text { sleep }\end{array}$ & $69(18.0 \%)$ & $37(20.1 \%)$ & $32(16.0 \%)$ & & $18(20.2 \%)$ & $9(16.4 \%)$ & $23(12.9 \%)$ & $19(300.6 \%)$ & \\
\hline & Both a and b & $172(44.8 \%)$ & $78(42.4 \%)$ & $94(47.0 \%)$ & & $39(43.8 \%)$ & $41(74.5 \%)$ & $65(36.5 \%)$ & $27(43.5 \%)$ & \\
\hline
\end{tabular}


Vinita Mary et al: Knowledge, Attitude And Practices Of Dental Students And Practitioners

\begin{tabular}{|c|c|c|c|c|c|c|c|c|c|c|}
\hline & Don't know & $25(6.5 \%)$ & $12(6.5 \%)$ & $13(6.5 \%)$ & & $5(5.6 \%)$ & $0(0.0 \%)$ & $17(9.6 \%)$ & $3(4.8 \%)$ & \\
\hline \multirow[b]{2}{*}{$\begin{array}{l}\text { Have you } \\
\text { undergone } \\
\text { conscious } \\
\text { sedation } \\
\text { yoursel1f } \\
\text { for your dental } \\
\text { procedure }\end{array}$} & Yes & $133(34.6 \%)$ & $77(41.8 \%)$ & $56(28.0 \%)$ & \multirow[b]{2}{*}{0.004} & $33(37.1 \%)$ & $8(14.5 \%)$ & $58(32.6 \%)$ & $34(54.8 \%)$ & \multirow[b]{2}{*}{0.000} \\
\hline & No & $251(65.4 \%)$ & $107(58.2 \%)$ & $144(72.0 \%)$ & & $56(62.9 \%)$ & $47(85.5 \%)$ & $120(67.4 \%)$ & $28(45.2 \%)$ & \\
\hline \multirow{5}{*}{$\begin{array}{l}\text { Which technique } \\
\text { of conscious } \\
\text { sedation is used } \\
\text { in dentistry }\end{array}$} & Oral & $86(22.4 \%)$ & $34(18.5 \%)$ & $52(26.0 \%)$ & \multirow{4}{*}{0.493} & $15(16.9 \%)$ & $4(7.3 \%)$ & $58(32.6 \%)$ & $9(14.5 \%)$ & \multirow{4}{*}{0.000} \\
\hline & Intravenous & $172(44.8 \%)$ & $88(47.8 \%)$ & $84(42.0 \%)$ & & $36(40.4 \%)$ & $30(54.5 \%)$ & $72(40.4 \%)$ & $34(54.8 \%)$ & \\
\hline & Inhalation & $79(20.6 \%)$ & $39(21.2 \%)$ & $40(20.0 \%)$ & & $26(29.2 \%)$ & $17(30.9 \%)$ & $26(14.6 \%)$ & $10(16.1 \%)$ & \\
\hline & $\begin{array}{l}\text { Intramuscula } \\
\mathbf{r}\end{array}$ & $30(7.8 \%)$ & $14(7.6 \%)$ & $16(8.0 \%)$ & & $9(10.1 \%)$ & $4(7.3 \%)$ & $10(5.6 \%)$ & $7(11.3 \%)$ & \\
\hline & Don't know & $17(4.4 \%)$ & $9(4.9 \%)$ & $8(4.0 \%)$ & & $3(3.4 \%)$ & $0(0.0 \%)$ & $12(6.7 \%)$ & $2(3.2 \%)$ & \\
\hline \multirow{4}{*}{$\begin{array}{l}\text { At which stage of } \\
\text { conscious } \\
\text { sedation dental } \\
\text { procedures are } \\
\text { carried out }\end{array}$} & Minimal & $169(44.0 \%)$ & $82(44.6 \%)$ & $87(43.5 \%)$ & \multirow{4}{*}{0.336} & $46(51.7 \%)$ & $21(38.2 \%)$ & $79(44.4 \%)$ & $23(37.1 \%)$ & \multirow{4}{*}{0.074} \\
\hline & Moderate & $167(43.5 \%)$ & $75(40.8 \%)$ & $92(46.0 \%)$ & & $35(39.3 \%)$ & $28(50.9 \%)$ & $70(39.3 \%)$ & $34(54.8 \%)$ & \\
\hline & Deep & $34(8.9 \%)$ & $21(11.4 \%)$ & $13(6.5 \%)$ & & $7(7.9 \%)$ & $6(10.9 \%)$ & $17(9.6 \%)$ & $4(6.5 \%)$ & \\
\hline & Don't know & $14(3.6 \%)$ & $6(3.3 \%)$ & $8(4.0 \%)$ & & $1(1.1 \%)$ & $0(0.0 \%)$ & $12(6.7 \%)$ & $1(1.6 \%)$ & \\
\hline \multirow[t]{5}{*}{$\begin{array}{l}\text { For which all } \\
\text { procedures } \\
\text { conscious } \\
\text { sedation can be } \\
\text { used }\end{array}$} & $\begin{array}{l}\text { General } \\
\text { dentistry } \\
\text { such as } \\
\text { restoration } \\
\text { \&scaling } \\
\end{array}$ & $80(20.8 \%)$ & $43(23.4 \%)$ & $37(18.5 \%)$ & \multirow{5}{*}{0.267} & $18(20.2 \%)$ & $5(9.1 \%)$ & $37(20.8 \%)$ & $20(32.3 \%)$ & \multirow[t]{5}{*}{0.002} \\
\hline & $\begin{array}{l}\text { Minor } \\
\text { surgery }\end{array}$ & $74(19.3 \%)$ & $35(19.0 \%)$ & $39(19.5 \%)$ & & $14(15.7 \%)$ & $5(9.1 \%)$ & $38(21.3 \%)$ & $17(27.4 \%)$ & \\
\hline & $\begin{array}{l}\text { Minor bone } \\
\text { fracture }\end{array}$ & $37(9.6 \%)$ & $20(10.9 \%)$ & $17(8.5 \%)$ & & $10(11.2 \%)$ & $7(12.7 \%)$ & $18(10.1 \%)$ & $2(3.2 \%)$ & \\
\hline & All the above & $177(46.1 \%)$ & $82(44.6 \%)$ & $95(47.5 \%)$ & & $46(51.7 \%)$ & $37(67.3 \%)$ & $74(41.6 \%)$ & $20(32.3 \%)$ & \\
\hline & Don't know & $16(4.2 \%)$ & $4(2.2 \%)$ & $12(6.0 \%)$ & & $1(1.1 \%)$ & $1(1.8 \%)$ & $11(6.2 \%)$ & $3(4.8 \%)$ & \\
\hline \multirow{4}{*}{$\begin{array}{l}\text { Conscious } \\
\text { sedation in dental } \\
\text { practice can be } \\
\text { given by }\end{array}$} & Anesthetist & $151(39.3 \%)$ & $71(38.6 \%)$ & $80(40.0 \%)$ & \multirow{4}{*}{0.983} & $33(37.1 \%)$ & $32(58.2 \%)$ & $55(30.9 \%)$ & $31(50.0 \%)$ & \multirow{4}{*}{0.000} \\
\hline & Dentist & $95(24.7 \%)$ & $45(24.5 \%)$ & $50(25.0 \%)$ & & $22(24.7 \%)$ & $3(5.5 \%)$ & $51(28.7 \%)$ & $19(30.6 \%)$ & \\
\hline & Both a and b & $126(32.8 \%)$ & $62(33.7 \%)$ & $64(32.0 \%)$ & & $34(38.2 \%)$ & $18(32.7 \%)$ & $64(36.0 \%)$ & $10(16.1 \%)$ & \\
\hline & Don't know & $12(3.1 \%)$ & $6(3.3 \%)$ & $6(3.0 \%)$ & & $0(0.0 \%)$ & $2(3.6 \%)$ & $8(4.5 \%)$ & $2(3.2 \%)$ & \\
\hline \multirow{4}{*}{$\begin{array}{l}\text { In which of the } \\
\text { conditions } \\
\text { conscious } \\
\text { sedation is } \\
\text { indicated }\end{array}$} & $\begin{array}{l}\text { Mild to } \\
\text { moderate } \\
\text { dental } \\
\text { anxiety }\end{array}$ & $179(46.6 \%)$ & $84(45.9 \%)$ & $950(47.5 \%)$ & \multirow{4}{*}{0.886} & $45(50.6 \%)$ & $29(52.7 \%)$ & $87(48.9 \%)$ & $18(29.0 \%)$ & \multirow[t]{4}{*}{0.009} \\
\hline & $\begin{array}{l}\text { Moderate to } \\
\text { severe dental } \\
\text { anxiety }\end{array}$ & $139(36.2 \%)$ & $70(38.0 \%)$ & $69(34.5 \%)$ & & $29(32.6 \%)$ & $19(34.5 \%)$ & $55(30.9 \%)$ & $36(58.1 \%)$ & \\
\hline & $\begin{array}{l}\text { Severe dental } \\
\text { anxiety }\end{array}$ & $45(11.7 \%)$ & $21(11.4 \%)$ & $24(12.0 \%)$ & & $11(12.4 \%)$ & $7(12.7 \%)$ & $21(11.8 \%)$ & $6(9.7 \%)$ & \\
\hline & Don't know & $21(5.5 \%)$ & $9(4.9 \%)$ & $12(6.0 \%)$ & & $4(4.5 \%)$ & $0(0.0 \%)$ & $15(8.4 \%)$ & $2(3.2 \%)$ & \\
\hline \multirow{4}{*}{$\begin{array}{l}\text { Under which age } \\
\text { conscious } \\
\text { sedation is } \\
\text { contraindicated }\end{array}$} & $\begin{array}{l}\text { Below } 6 \\
\text { months }\end{array}$ & $218(56.8 \%)$ & $108(58.7 \%)$ & $110(55.0 \%)$ & \multirow[t]{4}{*}{0.247} & $56(62.9 \%)$ & $35(63.6 \%)$ & $100(56.2 \%)$ & $27(43.5 \%)$ & \multirow{4}{*}{0.025} \\
\hline & $\begin{array}{l}\text { Below } 10 \\
\text { months }\end{array}$ & $70(18.2 \%)$ & $36(20.7 \%)$ & $32(16.0 \%)$ & & $16(18.0 \%)$ & $13(23.6 \%)$ & $26(14.6 \%)$ & $15(24.2 \%)$ & \\
\hline & Below 1 year & $65(16.9 \%)$ & $25(13.6 \%)$ & $40(20.0 \%)$ & & $13(14.6 \%)$ & $7(12.7 \%)$ & $30(16.9 \%)$ & $15(24.2 \%)$ & \\
\hline & Don't know & $31(8.1 \%)$ & $13(7.1 \%)$ & $18(9.0 \%)$ & & $4(4.5 \%)$ & $0(0.0 \%)$ & $22(12.4 \%)$ & $5(8.1 \%)$ & \\
\hline \multirow{4}{*}{$\begin{array}{l}\text { In which of the } \\
\text { conditions } \\
\text { conscious } \\
\text { sedation is } \\
\text { contraindicated }\end{array}$} & $\begin{array}{l}\text { Extreme } \\
\text { anxiety }\end{array}$ & $250(65.1 \%)$ & $119(64.7 \%)$ & $131(65.5 \%)$ & \multirow{4}{*}{0.184} & $65(73.0 \%)$ & $40(72.7 \%)$ & $110(61.8 \%)$ & $35(56.5 \%)$ & \multirow{4}{*}{0.002} \\
\hline & $\begin{array}{l}\text { Needle } \\
\text { phobia }\end{array}$ & $56(14.6 \%)$ & $27(14.7 \%)$ & $29(14.5 \%)$ & & $16(18.0 \%)$ & $8(14.5 \%)$ & $17(9.6 \%)$ & $15(24.2 \%)$ & \\
\hline & $\begin{array}{l}\text { Traumatic } \\
\text { procedure in } \\
\text { uncooperativ } \\
\text { e patient }\end{array}$ & $50(13.0 \%)$ & $29(15.8 \%)$ & $21(10.5 \%)$ & & $6(6.7 \%)$ & $6(10.9 \%)$ & $29(16.3 \%)$ & $9(14.5 \%)$ & \\
\hline & Don't know & $28(7.3 \%)$ & $9(4.9 \%)$ & $19(9.5 \%)$ & & $2(2.2 \%)$ & $1(1.8 \%)$ & $22(12.4 \%)$ & $3(4.8 \%)$ & \\
\hline
\end{tabular}

Table2 (a).Comparison of Knowledge and Practice of Conscious Sedation among Genders and Qualification 
Vinita Mary et al: Knowledge, Attitude And Practices Of Dental Students And Practitioners

\begin{tabular}{|c|c|c|c|c|c|c|c|c|c|c|}
\hline \multirow[t]{2}{*}{ Question } & \multirow[t]{2}{*}{ Options } & \multirow{2}{*}{$\begin{array}{l}\text { Total } \\
\mathbf{n}(\%)\end{array}$} & \multicolumn{2}{|c|}{ Gender } & \multirow{2}{*}{$\begin{array}{c}\mathbf{P} \\
\text { value }\end{array}$} & \multicolumn{4}{|c|}{ Qualification } & \multirow{2}{*}{$\begin{array}{c}P \\
\text { value }\end{array}$} \\
\hline & & & $\begin{array}{l}\text { Males } \\
\text { n }(\%)\end{array}$ & $\begin{array}{c}\text { Females } \\
\text { n }(\%)\end{array}$ & & $\begin{array}{l}\text { BDS } \\
\text { n }(\%)\end{array}$ & $\begin{array}{c}\text { MDS n } \\
(\%)\end{array}$ & $\begin{array}{c}\text { UG } \\
\text { student } \\
\text { n (\%) }\end{array}$ & $\begin{array}{l}\text { PG student } \\
\text { n }(\%)\end{array}$ & \\
\hline \multirow{3}{*}{$\begin{array}{l}\text { Is intravenous } \\
\text { conscious sedation } \\
\text { monitored } \\
\text { continuously }\end{array}$} & Yes & $237(61.7 \%)$ & $114(62.0 \%)$ & $123(61.5 \%)$ & \multirow{3}{*}{0.005} & $66(74.2 \%)$ & $37(67.3 \%)$ & $97(54.5 \%)$ & $37(59.7 \%)$ & \multirow{3}{*}{0.000} \\
\hline & No & $76(19.8 \%)$ & $46(25.0 \%)$ & $30(15.0 \%)$ & & $13(14.6 \%)$ & $16(29.1 \%)$ & $30(16.9 \%)$ & $17(27.4 \%)$ & \\
\hline & Don't know & $71(18.5 \%)$ & $24(13.0 \%)$ & $47(23.5 \%)$ & & $10(11.2 \%)$ & $2(3.6 \%)$ & $51(28.7 \%)$ & $8(12.9 \%)$ & \\
\hline \multirow{2}{*}{$\begin{array}{l}\text { Do you know the } \\
\text { armamentarium } \\
\text { used in conscious } \\
\text { sedation }\end{array}$} & Yes & $240(62.5 \%)$ & $118(64.1 \%)$ & $122(61.0 \%)$ & & $56(62.9 \%)$ & $41(74.5 \%)$ & $96(53.9 \%)$ & $47(75.8 \%)$ & \multirow[b]{2}{*}{0.003} \\
\hline & No & $144(37.5 \%)$ & $66(35.9 \%)$ & $78(39.0 \%)$ & & $33(37.1 \%)$ & $14(25.5 \%)$ & $82(46.1 \%)$ & $15(24.2 \%)$ & \\
\hline \multirow{4}{*}{$\begin{array}{l}\text { Which is the } \\
\text { fastest action drug } \\
\text { for conscious } \\
\text { sedation }\end{array}$} & Midazolam & $154(40.1 \%)$ & $69(37.5 \%)$ & $85(42.5 \%)$ & \multirow{4}{*}{0.633} & $34(38.2 \%)$ & $20(36.4 \%)$ & $79(44.4 \%)$ & $21(33.9 \%)$ & \multirow{4}{*}{0.001} \\
\hline & Benzodiazepam & $135(35.2 \%)$ & $70(38.0 \%)$ & $65(32.5 \%)$ & & $33(37.1 \%)$ & $25(45.5 \%)$ & $52(29.2 \%)$ & $25(40.3 \%)$ & \\
\hline & Barbiturates & $48(12.5 \%)$ & $24(13.0 \%)$ & $24(12.0 \%)$ & & $14(15.7 \%)$ & $7(12.7 \%)$ & $13(7.3 \%)$ & $14(22.6 \%)$ & \\
\hline & Don't know & $47(12.2 \%)$ & $21(11.4 \%)$ & $26(13.0 \%)$ & & $8(9.0 \%)$ & $3(5.5 \%)$ & $34(19.1 \%)$ & $2(3.2 \%)$ & \\
\hline \multirow{4}{*}{$\begin{array}{l}\text { Which gas is used } \\
\text { for conscious } \\
\text { sedation }\end{array}$} & Nitrous oxide & $301(78.4 \%)$ & $141(76.6 \%)$ & $160(80.0 \%)$ & \multirow{4}{*}{0.354} & $72(80.9 \%)$ & $51(92.7 \%)$ & $139(78.1 \%)$ & $39(62.9 \%)$ & \multirow{4}{*}{0.000} \\
\hline & Carbon dioxide & $34(8.9 \%)$ & $18(9.8 \%)$ & $16(8.0 \%)$ & & $11(12.4 \%)$ & $0(0.0 \%)$ & $11(6.2 \%)$ & $12(19.4 \%)$ & \\
\hline & Oxygen & $32(8.3 \%)$ & $19(10.3 \%)$ & $13(6.5 \%)$ & & $6(6.7 \%)$ & $2(3.6 \%)$ & $14(7.9 \%)$ & $10(16.1 \%)$ & \\
\hline & Don't know & $17(4.4 \%)$ & $6(3.3 \%)$ & $11(5.5 \%)$ & & $0(0.0 \%)$ & $2(3.6 \%)$ & $14(7.9 \%)$ & $1(1.6 \%)$ & \\
\hline \multirow{4}{*}{$\begin{array}{l}\text { How long does } \\
\text { conscious sedation } \\
\text { last }\end{array}$} & 2-8 hours & $203(52.9 \%)$ & $99(53.8 \%)$ & $104(52.0 \%)$ & \multirow{4}{*}{0.094} & $48(53.9 \%)$ & $42(76.4 \%)$ & $84(48.3 \%)$ & $27(43.5 \%)$ & \multirow{4}{*}{0.000} \\
\hline & 1-4 hours & $106(27.6 \%)$ & $55(29.9 \%)$ & $51(25.5 \%)$ & & $30(33.7 \%)$ & $9(16.4 \%)$ & $43(24.2 \%)$ & $24(38.7 \%)$ & \\
\hline & $2-5$ hours & $37(9.6 \%)$ & $19(10.3 \%)$ & $18(9.0 \%)$ & & $6(6.7 \%)$ & $4(7.3 \%)$ & $17(9.6 \%)$ & $10(16.1 \%)$ & \\
\hline & Don't know & $38(9.9 \%)$ & $11(6.0 \%)$ & $27(13.5 \%)$ & & $5(5.6 \%)$ & $0(0.0 \%)$ & $32(18.0 \%)$ & $1(1.6 \%)$ & \\
\hline \multirow{4}{*}{$\begin{array}{l}\text { What is the } \\
\text { complications of } \\
\text { conscious sedation }\end{array}$} & Hypoxia & $252(65.6 \%)$ & $112(60.9 \%)$ & $140(70.0 \%)$ & \multirow{4}{*}{0.029} & $60(67.4 \%)$ & $40(72.7 \%)$ & $119(66.9 \%)$ & $33(53.2 \%)$ & \multirow{4}{*}{0.000} \\
\hline & Patient retrieval & $64(16.7 \%)$ & $36(9.6 \%)$ & $28(14.0 \%)$ & & $19(21.3 \%)$ & $8(14.5 \%)$ & $17(9.6 \%)$ & $20(32.3 \%)$ & \\
\hline & Anxiety & $38(9.9 \%)$ & $22(12.0 \%)$ & $16(8.0 \%)$ & & $8(9.0 \%)$ & $6(10.9 \%)$ & $19(10.7 \%)$ & $5(8.1 \%)$ & \\
\hline & Don't know & $30(7.8 \%)$ & $14(7.6 \%)$ & $16(8.0 \%)$ & & $2(2.2 \%)$ & $1(1.8 \%)$ & $23(12.9 \%)$ & $4(6.5 \%)$ & \\
\hline Is oral sedation & Yes & $231(60.2 \%)$ & $110(59.8 \%)$ & $121(60.5 \%)$ & & $59(66.3 \%)$ & $43(78.2 \%)$ & $92(51.7 \%)$ & $37(59.7 \%)$ & \\
\hline safer than other & No & $79(20.6 \%)$ & $45(24.5 \%)$ & $34(17.0 \%)$ & & $14(15.7 \%)$ & $8(14.5 \%)$ & $36(20.2 \%)$ & $21(33.9 \%)$ & \\
\hline modes of sedation & Don't know & $74(19.3 \%)$ & $29(15.8 \%)$ & $45(22.5 \%)$ & 0.088 & $16(18.0 \%)$ & $4(7.3 \%)$ & $50(28.1 \%)$ & $4(6.5 \%)$ & 0.000 \\
\hline Is $\quad$ conscious & Yes & $92(24.0 \%)$ & $46(25.0 \%)$ & $46(23.0 \%)$ & & $30(33.7 \%)$ & $6(10.9 \%)$ & $39(21.9 \%)$ & $17(27.4 \%)$ & \\
\hline sedation safe & No & $249(62.8 \%)$ & $114(62.0 \%)$ & $127(63.5 \%)$ & & $51(57.3 \%)$ & $43(78.2 \%)$ & $113(63.5 \%)$ & $34(54.8 \%)$ & 0.032 \\
\hline during pregnancy & Don't know & $51(13.3 \%)$ & $24(13.0 \%)$ & $27(13.5 \%)$ & 0.900 & $8(9.0 \%)$ & $6(10.9 \%)$ & $26(14.6 \%)$ & $11(17.7 \%)$ & \\
\hline Can you eat or & Yes & $173(45.1 \%)$ & $83(45.1 \%)$ & $90(45.0 \%)$ & & $46(51.7 \%)$ & $30(54.5 \%)$ & $71(39.9 \%)$ & $26(41.9 \%)$ & \\
\hline drink before and & No & $155(40.4 \%)$ & $74(40.2 \%)$ & $81(40.5 \%)$ & 0.988 & $33(37.1 \%)$ & $21(38.2 \%)$ & $75(42.1 \%)$ & $26(41.9 \%)$ & \\
\hline $\begin{array}{l}\text { after conscious } \\
\text { sedation }\end{array}$ & Don't know & $56(14.6 \%)$ & $27(14.7 \%)$ & $29(14.5 \%)$ & & $10(11.2 \%)$ & $4(7.3 \%)$ & $32(18.0 \%)$ & $10(16.1 \%)$ & 0.250 \\
\hline $\begin{array}{l}\text { How many hours } \\
\text { should you not eat } \\
\text { and drink before }\end{array}$ & $\begin{array}{l}2 \text { hours for } \\
\text { fluids and } 6 \\
\text { hours for solids }\end{array}$ & $184(47.9 \%)$ & $85(46.2 \%)$ & $99(49.5 \%)$ & 0.308 & $53(59.6 \%)$ & $24(43.6 \%)$ & $77(43.3 \%)$ & $30(48.4 \%)$ & 0.000 \\
\hline & $\begin{array}{l}6 \text { hours for } \\
\text { fluids and } 6 \\
\text { hours for solids }\end{array}$ & $106(27.6 \%)$ & $58(31.5 \%)$ & $48(24.0 \%)$ & & $21(23.6 \%)$ & $23(41.8 \%)$ & $41(23.0 \%)$ & $21(33.9 \%)$ & \\
\hline & $\begin{array}{l}3 \text { hours for } \\
\text { fluids and } 5 \\
\text { hours for solids }\end{array}$ & $30(7.8 \%)$ & $15(8.2 \%)$ & $15(7.5 \%)$ & & $7(7.9 \%)$ & $3(5.5 \%)$ & $13(7.3 \%)$ & $7(11.3 \%)$ & \\
\hline & Don't Know & $64(16.7 \%)$ & $26(14.1 \%)$ & $38(19.0 \%)$ & & $8(9.0 \%)$ & $5(9.1 \%)$ & $47(26.4 \%)$ & $4(6.4 \%)$ & \\
\hline Do you aware of & Yes & $235(61.2 \%)$ & $119(64.7 \%)$ & $116(58.0 \%)$ & 0.180 & $53(59.6 \%)$ & $34(61.8 \%)$ & $96(53.9 \%)$ & $52(83.9 \%)$ & 0.001 \\
\hline $\begin{array}{l}\text { the crash cart } \\
\text { equipment for } \\
\text { conscious sedation }\end{array}$ & No & $149(38.8 \%)$ & $65(35.3 \%)$ & $84(42.0 \%)$ & & $36(40.4 \%)$ & $21(38.2 \%)$ & $82(46.1 \%)$ & $10(16.1 \%)$ & \\
\hline
\end{tabular}

Table 2 (b) Comparison of Knowledge and Practice of Conscious Sedation among Genders and Qualification

\section{DISCUSSION}

Management of pediatric patients during dental procedures plays a crucial role to establish ideal communication, eliminate fear and anxiety, build trust between the dentist, child, and parent, promote and enhance child's positive attitude towards dental health by Volume No: 8, Issue No: 3 successfully incorporating behavioral guidance thus providing quality oral health care. In the present study it was observed more than half of the study participants learned about conscious sedation (CS) through dental curriculum $(50.8 \%)$ comparable to study by Thomas $\mathrm{P}$ et $\mathrm{al}^{16}$ where the majority of the participants were aware of 
Vinita Mary et al: Knowledge, Attitude And Practices Of Dental Students And Practitioners

conscious sedation $(80.1 \%)$ through their curriculum yet over $69.4 \%$ among them also preferred short certified courses to enhance their practice in conscious sedation $^{1,3,17,18}$.

It is evident that advanced behavior guidance techniques such as conscious sedation, General anesthesia (GA), and protective stabilization were commonly taught and practiced in postgraduate/masters pediatric dental training programs. A study done by Monisha K et al (90\%), reported that more than half of the pediatric dental surgeons reported the use of conscious sedation over $\mathrm{GA}^{18}$ comparable to the present study where $46.1 \%$ considered conscious sedation over GA for all dental procedures like restoration, scaling, minor bone fractures and minor surgeries. A higher prevalence might be attributed to observations done only on dental professionals in the present study whereas previous study involved parents as well as other health care professionals also. In contrast, some studies suggest that dental surgeons working in hospitals preferred the utilization of $\mathrm{GA}^{18,19,20}$.

In our study, $44.8 \%$ preferred intravenous conscious sedation over oral sedation which is almost similar to the study by Tingey et al $(49.8 \%){ }^{21}$ but much higher than the study by Sivakumar $\mathrm{N}$ et al $(3 \%)^{22}$. This variation can be due to case preferences as sedation in children is different from the sedation methods in adults. Cunha et al stated that sedation in children is often used to control behavior and permit safe completion of the dental procedure and it depended on chronological as well as developmental age of the child ${ }^{1,18,23}$.

In this present study, the participants preferred to treat patients in minimal or moderate conscious sedation state. But few studies suggest that moderate sedation was comfortable to diagnose and treat uncooperative or anxious patients, decreased the pain and discomfort compared to minimum sedation and also maximized the effectiveness of amnesia, and controlling the anxiety, movement, and behavior of the child during dental procedures $23,24,25,26$.

We observed that $56.8 \%$ of the participants considered that conscious sedation was contraindicated in children below 6 months of age and with extreme fear and anxiety. Likewise, Veeramachaneni et $\mathrm{al}^{24}$ states that the Council of European Dentist and AAPD (American association of Pediatric dentistry) has recommended that this technique of behavior control should not be administered in severely compromised patients and in children less than 1 year $^{13}$.
In the present study, more than half of the study participants $78.4 \%$ preferred Nitrous oxide sedation which is similar to study by Sivakumar $\mathrm{N}$ et al $(72 \%)^{22}$ and Wali et al $(73 \%)^{1}$. These studies have shown that care must be taken when Nitrous oxide sedation is used in addition to other sedatives where the chances of attaining deep sedation in a short span of time are relatively high ${ }^{1}$.

In the present study, 39.3\% preferred that general anesthetist should be present during conscious sedation technique. In a study by Thomas $\mathrm{P}$ et $\mathrm{al}^{16}$, it was observed that the dentist experienced difficulties in managing sedation in the absence of anesthesiologists resulting in anxiety and forced and hasty completion of the treatment. However, in presence of anesthesiologists, dentist failed to experience such nervousness thus providing quality dental treatment while the anesthesiologists took care of the vital signs and related complications. Also, AAPD recommends that Pediatric dentists must be well-qualified and skilled for incorporating specific training in their practice to integrate a multidisciplinary team approach which permits the recognition of the need of general anesthesia thus providing

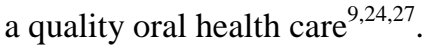

In the present study, $40.1 \%$ recommended midazolam as a fast acting drug for conscious sedation which is in contrast to the study by Sivakumar $\mathrm{N}$ et al $(76 \%)^{2}$. It can be observed that higher preference of midazolam might be due to its short duration of action with potent anxiolytic and anticonvulsant property over other drugs such as propofol. Also, midazolam has lower incidence of complications like nausea, vomiting and prolonged discharge time when compared to other drugs given orally and/or nasally ${ }^{28,29,30}$.

About $60.2 \%$ opted for oral route as the safer mode of sedation which is similar to study by Perumal $\mathrm{K}$ et al $(58 \%)^{8}$. Most of the study participants believed oral sedatives were easier to administer, required less expertise and skill, avoided needle usage and adequate sedation was achieved with minimal or no complications ${ }^{31}$.

\section{RECOMMENDATION}

It is recommended that dental curriculum should include guidelines and advanced sedation techniques to train the dental students at the undergraduate level. Subsequently dental practitioners should be encouraged to attend workshops, continuing dental education programs on conscious sedation technique by experts in the field to improve their skills and confidence. 
Vinita Mary et al: Knowledge, Attitude And Practices Of Dental Students And Practitioners

\section{CONCLUSION}

The present clearly shows lacunae in adequate knowledge, attitude and practices towards conscious sedation among dental practitioners. One must be also aware of patient related factors like perceptions, preferences and treatment needs nonetheless managing an uncooperative or anxious child is always challenging that requires skill and expertise with sufficient knowledge and awareness. The results of this study thus provide an insight on need for strategies to provide knowledge and incorporate positive attitude among the Dental professionals to offer effective conscious sedation in children necessitating oral health care.

\section{FINANCIAL SUPPORT AND SPONSORSHIP}

Nil

\section{CONFLICTS OF INTEREST}

There are no conflicts of interest.

\section{REFERENCES}

1. Wali A, Siddiqui TM, Khan R, Batool K. Knowledge, Attitude, and Practices of Dental Surgeons in managing Child Patients. Int J Clin Pediatr Dent. 2016 Oct-Dec; 9(4):372-378.

2. Merdad L, El-Housseiny AA. Do children's previous dental experience and fear affect their perceived oral health-related quality of life (OHRQoL). BMC Oral Health 2017 Dec; 17(1):19.

3. Bhandari R, Thakur S, Singhal P, Chauhan D, Jayam C, Jain T. Parental awareness, knowledge, and attitude toward conscious sedation in North Indian children population: A questionnaire-based study. Indian J Dent Res 2018;29:693-7

4. Anthonappa RP, Ashley PF, Bonetti DL, Lombardo G, Riley P. Non-pharmacological interventions for managing dental anxiety in children. The Cochrane Database of Systematic Reviews. 2017 Jun; 2017(6).

5. Costa LR, Bendo CB, Daher A, Heidari E, Rocha RS, Moreira AP, Moura LS, Banerjee A, Newton JT, Hosey MT. A curriculum for behaviour and oral healthcare management for dentally anxious children-Recommendations from the Children Experiencing Dental Anxiety: Collaboration on Research and Education (CEDACORE). International journal of paediatric dentistry. 2020 Sep; 30(5):556-69.
6. Armfield JM, Heaton LJ. Management of fear and anxiety in the dental clinic: a review. Australian dental journal. 2013 Dec; 58(4):390-407.

7. American Academy of Pediatric Dentistry. Guideline on use of Anesthesia Personnel in the Administration of Office-based Deep Sedation/General Anesthesia to the Pediatric Dental Patient. Pediatr Dent. 2013; 34(5):170-2.

8. Perumal K, Santhosh Kumar M.P. Knowledge and Attitude among Dental Undergraduate Students towards Sedation in Dental Practice. Int. J. Pharm. Sci. Rev. Res., 44(1), May - June 2017; 62-65

9. Silva CC, Lavado C, Areias C, Mourão J, Andrade DD. Conscious sedation vs general anesthesia in pediatric dentistry-a review. Medical Express. (São Paulo, online) Feb 2015: 2(1).

10. Dougherty N. The dental patient with special needs: a review of indications for treatment under general anesthesia. Special Care in Dentistry. 2009 Jan; 29(1):17-20.

11. Baghdadi ZD, Jbara S, Muhajarine N. Children's drawing as a projective measure to understand their experiences of dental treatment under general anesthesia. Children. $2020 \mathrm{Jul} ;$ 7(7):73.

12. Attri JP, Sharan R, Makkar V, Gupta KK, Khetarpal R, Kataria AP. Conscious sedation: Emerging trends in pediatric dentistry. Anesthesia, essays and researches. 2017 Apr; 11(2):277.

13. Hallonsten AL, Jensen B, Raadal M, Veerkamp J, Hosey MT, Poulsen S. EAPD guidelines on sedation in paediatric dentistry. European Academy of Paediatric Dentistry Im Internet: http://www eapd gr/dat/5CF03741/file pdf Stand. 2013; 7:12.

14. Rai K, Hegde A, Goel K. Sedation in uncooperative children undergoing dental procedures: A comparative evaluation of midazolam, propofol and ketamine. Journal of Clinical Pediatric Dentistry. 2007 Sep 1; 32(1):1-4.

15. Heard C, Smith J, Creighton P, Joshi P, Feldman D, Lerman J. A comparison of four sedation techniques for pediatric dental surgery. Pediatric Anesthesia. 2010 Oct; 20(10):924-30

16. Princy Thomas, Bhavna Dave, Seema Bargale, Poonacha, KS., Parth Joshi and Brijesh Tailor,2018. "Perception among dentists of gujarat state regarding the use of conscious sedation in pediatric dental practice " International Journal of Current Research in Life Sciences, 7, (05), 20042009. 
Vinita Mary et al: Knowledge, Attitude And Practices Of Dental Students And Practitioners

17. Adair SM, Rockman RA, Schafer TE, Waller JL. Survey of behavior management teaching in pediatric dentistry advanced education programs. Pediatr Dent. $2004 \quad$ Mar-Apr; 26(2):151-158.

18. Monisha.K et al. Knowledge, Attitude And Practice On Conscious sedation In Children Among Dental Practitioners', International Journal of Current Advanced Research, 2017; 06(04), 3033-3036.

19. Abushal MS, Adenubi JO. The use of behavior management techniques by dentists in Saudi Arabia: a survey. Saudi Dent J. 2000; 12(3):129134.

20. . McKnight-Hanes C, Myers DR, Dushku JC, Davis HC. The use of behavior management techniques by dentists across practitioner type, age, and geographic region. Pediatr Dent. 1993 JulAug; 15(4):267-271.

21. Tingey BT, Clark SH, Humbert LA, et al. Use of Intravenous Sedation in Periodontal Practice: A National Survey. J Periodontol. 2012;83:830-835.

22. Sivakumar N, Dhanraj Ganapathy, Kiran Kumar, Pandurangan and Ashok Velayudhan ,Awareness on Commonly used Sedatives Hypnotic in Dental Practices among Dental Student ; JPRI. 2020 32(18): 188-196.

23. Wilson S, Farrell K, Griffen A, Coury D. Conscious sedation experiences in graduate pediatric dentistry programs. Pediatric dentistry. 2001 Jul 1; 23(4):307-14.

24. Veeramachaneni D, Dande D, Mohan D, Ajay T, Khan D, Muzammil M, Verma DS, Parhad D. Case Preferences In Choosing Consious Sedation Versus General Anesthesia Amongst Pediatric
Dentists: A Research Survey. European Journal of Molecular \& Clinical Medicine. 2021 Jan 18; 8(1):1252-7.

25. Eaton JJ, McTigue DJ, Fields HW, Beck FM. Attitudes of contemporary parents toward behavior management techniques used in pediatric dentistry. Pediatric Dentistry. 2005; 27(2):107-113.

26. Gozal D, Drenger B, Levin PD, Kadari A, Gozal Y. A pediatric sedation/anesthesia program with dedicated care by anesthesiologists and nurses for procedures outside the operating room. J Pediatr. 2004; 145(1):47-52.

27. Silay E., et al. "Conscious sedation with midazolam for dental procedures be an alternative to general anesthesia?" Nigerian Journal of Clinical Practice 16.2: 2013; 211-215.

28. Averley PA, Girdler NM, Bond S, Steen N, Steele J. A randomised controlled trial of pediatric conscious sedation for dental treatment using intravenous midazolam combined with inhaled nitrous oxide or nitrous oxide/sevoflurane. Anesthesia. 2004; 59:844-52.

29. Jensen B, Matsson L. Oral versus rectal midazolam as a pre-anaesthetic sedative in children receiving dental treatment under general anaesthesia. Acta Paediatr. 2002; 91:920-5.

30. Arya VS, Damle SG. Comparative evaluation of midazolam and propofol as intravenous sedative agents in the management of unco-operative children. J Indian Soc Pedod Prev Dent. 2002; 20:6-8.

31. Alzahrani AM, Wyne AH. Use of Oral Midazolam Sedation in Pediatric Dentistry: A Review. Pakistan Oral \& Dental Journal. 2012 Dec 1; 32(3). 